\title{
Clinical Population
}

Pharmacokinetics/Pharmacodynamics Protocol of

\section{Alkaloids from Leaf of Alstonia scholaris on Acute Bronchitis Patients}

\section{Rui Li}

China Academy of Chinese Medical Sciences Xiyuan Hospital

\section{Wan-Tong Zhang}

China Academy of Chinese Medical Sciences Xiyuan Hospital

\section{Yun-Li Zhao}

China Academy of Chinese Medical Sciences Xiyuan Hospital

\section{Ming-Yue Sun}

China Academy of Chinese Medical Sciences Xiyuan Hospital

\section{Mao-Rong Fan}

China Academy of Chinese Medical Sciences Xiyuan Hospital

\section{Xiao-Dong Luo}

Chinese academy of sciences

Rui Gao (Dxyyyg@126.com )

Xin-Gang Li

Beijing friendship hospital

\section{Study protocol}

Keywords: Capsule of alkaloids from leaf of A. scholaris (CALAS); Acute tracheobronchitis; Population pharmacokinetics/pharmacodynamics

Posted Date: September 2nd, 2019

DOl: https://doi.org/10.21203/rs.2.13848/v1

License: (c) (1) This work is licensed under a Creative Commons Attribution 4.0 International License. Read Full License 


\section{Abstract}

Background Acute tracheobronchitis is the acute tracheobronchial mucosal inflammation caused by biological, physical, chemical stimulation, and accompanied by symptoms of cough and expectoration. The capsule of alkaloids from leaf of Alstonia scholaris (CALAS), an effective part riches in alkaloids, has the antiviral, antibacterial, anti-inflammatory, expectorant, anti-tussive, anti-asthmatic, immunoregulation effects. However, whether the clinical indexes could be improved by the same ingredients and pathways after oral administration, and the changes in the relationship between ingredients and pharmacodynamics indexes, still need further clinical verification.Methods/design This is a prospectively planned, blinded, placebo-controlled, parallel-grouped clinical trial with aggregated population pharmacokinetics/pharmacodynamics (PPK/PD) data. 55 subjects will be randomly allocated into four arms, specifically, 10 of the 55 subjects were selected randomly for the placebo arm who will be orally administered with placebo (Tid), and 45 subjects were randomly assigned to CALAS treatment group (20 mg, $40 \mathrm{mg}, 80 \mathrm{mg}$ Tid, 15 subjects per group). The medication, cough and phlegm, body temperature of every subject should be recorded daily during treatment. Each subject will be collected 3-4 blood samples at the following points for PPK/PPD parameters analysis. Blood samples will be acquired pre-dose $(0 \mathrm{~h})$ and post-dose at $15 \mathrm{~min}, 40 \mathrm{~min}, 1 \mathrm{~h}, 1.5 \mathrm{~h}, 2 \mathrm{~h}, 3 \mathrm{~h}, 4 \mathrm{~h}, 6 \mathrm{~h}, 8 \mathrm{~h}, 12 \mathrm{~h}, 24 \mathrm{~h}, 30 \mathrm{~h}, 48 \mathrm{~h}$ after last dosing. All subjects will receive a laboratory examination and efficacy evaluation on day 8.Discussion A new integrating strategy to explore relationship among drug ingredients, action pathway and clinical efficacy will be established through this study. We aim to explore the mechanism of CALAS in treatment of acute bronchitis on the premise of definite active ingredients and reliable clinical efficacy. It is difficult to be accepted by patients since classical pharmacokinetics (PK) research adopts intensive sampling method, meanwhile, it cannot quantify the variability of PK parameters (Intra-individual variation, interindividual variation and weekly variation). Moreover, extrapolation and prediction of dosage regimen between different species and populations cannot be realized. Therefore, PPK/PPD method, usually taking advantage of sparse data (3-5 time points sampling per patient), which be selected to find out the measurable factors of pathology and physiology that lead to the change of dose-concentration, and guide reasonable dose adjustment to achieve the optimal clinical effects.

\section{Background}

Acute tracheobronchitis is the acute tracheobronchial mucosal inflammation caused by biological, physical, chemical stimulation, and accompanied by the symptoms of cough and expectoration ${ }^{[1]}$. Nowadays, 14 drugs, the most commonly used in clinics for acute bronchitis treatment, are antibacterial and anti-inflammatory drugs, however, hospital-acquired infection caused by antibiotics abuse has become a potential safety problem, therefore, strict restrictions on antibiotic use have been promulgated by Ministry of Health of the People's Republic of China. In addition, the incidence rate of respiratory disease has risen sharply with the increasing deterioration of air quality, so that the research and development of respiratory drugs will certainly become a hotspot. 
The leaves of $A$. scholaris, commonly grown in Yunnan, Guangdong and Guangxi Province of China, have been traditionally used for the treatment of respiratory diseases. The capsule of alkaloids from leaf of $A$. scholaris (CALAS), an effective part riches in alkaloids, has the antiviral, antibacterial, anti-inflammatory, expectorant, anti-tussive, anti-asthmatic, immunoregulation effect ${ }^{[2]}$. CALAS has the function of treating cough, phlegm and wheeze, relieving bronchial mucosal swelling and expanding bronchi, moreover, it can achieve the combined effect of antibiotics, expectorant and anti-tussive medicine to some extent, control infection through immunological enhancement, and slow down the onset progress of acute bronchitis fundamentally.

Preclinical pharmacodynamics study indicated that the main compounds--Picrinine, Vallesamine, Scholaricine, 19-Epi-scholaricine showed therapeutic effects on airway inflammation, asthma, postinfectious cough, by decreasing the number of inflammatory cells and the content of cytokines such as neutrophils, eosinophils, IgE and Eotaxin, improving the balance of oxidation and antioxidation ${ }^{[3-5]}$.

Prior network pharmacology study predicted that CALAS could directly act on 8 acute bronchitis targets ${ }^{[6]}$ : ALB (Serum albumin), AR (Androgen receptor), GSTP1 (Glutathione S-transferase P), HMOX1 (Heme oxygenase 1), MMP13 (Collagenase 3), KDR (Vascular endothelial growth factor receptor 2), PDE4B (cAMP-specific 3,5-cyclic phosphodiesterase 4B) and PDE4D (cAMP-specific 3,5-cyclic phosphodiesterase 4D). Among of them, ALB, AR, HMOX1 and KDR are relatively important targets in the molecular biological network of acute bronchitis. compared with other targets. And four compunds of CALAS have 3 to 5 different targets. All the results indicated that the CALAS played an important role in the treatment of acute bronchitis and the potential pharmacological mechanisms were associated with anti-inflammatory, regulating endocrine and immune function and improving lung function ${ }^{[6]}$.

\section{Population PK/PD}

The population PK/PD model comprising pharmacokinetics (PK) and pharmacodynamics (PD), can precisely describe the discipline of time - concentration - effects and evaluate the relationship between drug concentration and efficacy ${ }^{[7]}$. Traditional PK research usually adopts intensive sampling method, which is hard to be accepted by patients, moreover individual variability of pharmacokinetics parameter couldn't be quantified ${ }^{[8]}$. Therefore, population pharmacodynamics is the optimum method to determine the population value and variability of PK/PD parameter as well as the influence of fixed effect. Population pharmacodynamics research take advantage of sparse data, usually 3-5 time points sampling per patient, to find out the measurable pathological and physiological factors which lead to the change of dose-concentration, and guide reasonable dose adjustment to achieve the optimal clinical effect $^{[9]}$.

\section{Why is this study needed now?}

Although the preclinical research indicated good therapeutic effect of CALAS on acute bronchitis, the question still need further clinical verification that whether the same result could be translated to human, 
whether the clinical indexes could be improved by the same ingredients and pathway after oral administration, furthermore, how dose the relationship between ingredients and pharmacodynamics indexes change? In this study, the potential pharmacological mechanism will be explored by network pharmacology research. Furthermore, the research of the PPK/PPD on the patients, setting these targets as the PD index, will be conducted to obtain the best efficacy dosage and guide rational drug use. The scientific data would be provided for clinical physicians to guide rational use of CALAS with the implementation of this study, meantime the new ideas and methods for scientific evaluation of traditional Chinese medicine (TCM) material-effect relationship would be presented.

\section{Objectives}

Establish PPK/PD model of capsule of alkaloids from leaf of $A$. scholaris (CALAS) in patients with acute bronchitis and provide effective evidences for the prediction of blood concentration and individualized administration of CALAS.

\section{Study design}

This is a prospectively planned, blinded, placebo-controlled, parallel-grouped clinical trial with aggregated PPK/PD data. 55 subjects will be randomly allocated into four arms, specifically, 10 of the 55 subjects were selected randomly for the placebo arm who will be orally administered with placebo (Tid), and 45 subjects were randomly assigned to CALAS treatment group $(20 \mathrm{mg}, 40 \mathrm{mg}, 80 \mathrm{mg}$ Tid, 15 subjects per group). Each subject will be randomized to a single dose. The medication, cough and phlegm, body temperature of every subject should be recorded daily during treatment. Each subject will be collected 34 blood samples at the following timepointsfor PPK-PPD parameter analysis. Blood samples will be acquired pre-dose (0h) and post-dose administration at $15 \mathrm{~min}, 40 \mathrm{~min}, 1 \mathrm{~h}, 1.5 \mathrm{~h}, 2 \mathrm{~h}, 3 \mathrm{~h}, 4 \mathrm{~h}, 6 \mathrm{~h}, 8 \mathrm{~h}, 12$ $h, 24 h, 30 h, 48 \mathrm{~h}$ after administration. All subjects will receive a laboratory examination and efficacy evaluation on day 8 (Table 1).

\section{Randomization and blinding}

According to simple randomization method, 55 subjects are randomized into four groups: placebo: 20 mg: 40 mg: 80 mg group = 10: 15: 15: 15, Furthermore, random coding and drug coding are generated by SAS 9.4 software.

Double blind design is adopted in this trial, that is blind codes that recording drug number and corresponding treatment group are preserved by principal investigator and statistician. Blind codes, folded three times, and sealed in opaque envelopes, which the paste margin is stamped. The blind codes should not be opened during the trial.

\section{Control}

Placebo control is adopted in this trial. 


\section{Sample size}

There are 4 groups in this trial, i.e. placebo group, $20 \mathrm{mg}$ group, $40 \mathrm{mg}$ group and $80 \mathrm{mg}$ group, 55 subjects will be enrolled in the trial, 10 subjects will be randomized in placebo group, 15 subjects will be respectively randomized in $20 \mathrm{mg}, 40 \mathrm{mg}$ and $80 \mathrm{mg}$ group.

\section{Study setting}

The trial will be conducted in outpatient and Clinical Pharmacology Institution (CPI) at Xiyuan Hospital, China Academy of Chinese Medical Sciences (CACMS). The respiratory physicians will pre-evaluate the patients according to the inclusion and exclusion criteria as follows, and recommend the suitable patients to the study investigators in CPI. All patients voluntarily joined this study with informed consents, and then fundmental indexes including the inquiry, vital signs and physical examination, laboratory examination, ECG and chest radiography will be conducted. The result will be estimated according to the inclusion and exclusion criteria. Eligible subjects will take CALAS on the next morning for 7 days, and participants will return to hospital for safety and effective evaluation on the $8^{\text {th }}$ day. The blood samples will be sent to Shenyang Pharmaceutical University for analysis, and the PPK-PPD data will be analyzed by Beijing Friendship Hospital, Capital Medical University.

\section{Patient and Public Involvement}

Patients involvement will be conducted in department of respiratory. The respiratory physicians will preevaluate the patients according to the inclusion and exclusion criteria as follows, and recommend the suitable patients to the study investigators in $\mathrm{CPI}$.

\section{Study participants}

\section{Inclusion criteria}

Participants must fulfill the following inclusion criteria: (1) Aging from 18 to 65 years; (2) In line with the diagnostic criteria of acute trachea-bronchitis; (3) Being less than $48 \mathrm{~h}$ of disease course; (4) Being above 5 scores of Bronchitis severity score(BSS); (5) Being informed of the study and voluntarily sign an informed consent.

\section{Exclusion criteria}

Subjects who meet any of the following will be excluded: (1) The upper limit of leucocyte exceeds $11 * 10^{9} / \mathrm{L}$ or the neutrophil exceeds $80 \%$; (2) With severe heart disease (acute myocardial infarction or acute myocardial infarction in 6 months), severe cardiopulmonary dysfunction, mental or physical disorders, severe diabetes and immunodeficiency; (3) With severe primary diseases, such as liver and renal hematopoietic system damage, liver function (ALT $\geq 2 \times U L N, A S T \geq 2 \times U L N$ ), kidney function ( $C r$ $>1.0 \times \mathrm{ULN}$ ); (4) With drug allergy history or with allergic constitution, or at least two drug allergies; (5) Pregnant, breast-feeding, and menstruating women, as well as women planning pregnancy within 3 
months; (6) Patients who participated in other clinical trials in the last 3 months; (7) Those who cannot take medicine as prescribed by the doctor;(8) People with poor compliance or unsuitability for this clinical trial by investigator's judgement.

\section{Termination criteria}

\section{Subject termination}

1. The investigator may suspend the subject for ethical consideration.

2. The investigator may suspend the subject if a serious adverse event (SAE) appears.

3. The investigator believes that it is beneficial for subject to withdraw from the trial.

\section{Trial termination}

The investigator finds safety problems.

1. It is difficult to realize the purpose of the study due to serious deviation.

2. The project management unit asks the termination of trial.

\section{Interventions}

\section{(1) medicine}

CALAS is a commercial formulation of $A$. scholaris leaf with functions of clearing heat, eliminating phlegm, diffusing the lung and relieving cough due to colds and acute bronchitis. Subjects are required to accurately record the medication, cough condition and temperature during the first 7 days, and taking CALAS with $250 \mathrm{ml}$ water under the supervision of the investigators on days 8 , The administration time is recorded as " 0 " hour.

(2) Dosage regimen:

- $20 \mathrm{mg}$ arm: 15 subjects take CALAS (20 mg, Tid) for 7 days.

- 40mg arm: 15 subjects take CALAS (40 mg, Tid) for 7 days.

- 80mg arm: 15 subjects take CALAS ( $80 \mathrm{mg}$, Tid) for 7 days.

- Placebo arm: 10 subjects take placebo (Tid) for 7 days.

(3) Combination therapy

$\triangle$ Antibiotics, western or Chinese medicines with function of relieving cough and reducing sputum are forbidden during the entire trial period, if the patient needs to use the medicine, and the rationality should be evaluated by respiratory physician in advance. All combination should be documented in the case report form (CRF). 
Q If AE happens, the necessary treatment should be performed according to the subject's condition, and continuous monitoring should be conducted until the AE restored to a medically acceptable level. All combination and treatment should be documented in CRF.

Sample collection

PK sampling

3 to 4 blood samples of each subject will be collected from the elbow vein and the total sampling need to cover the time-points of $0 \mathrm{~h}$ (before last dosing), and $15 \mathrm{~min}, 40 \mathrm{~min}, 1 \mathrm{~h}, 1.5 \mathrm{~h}, 2 \mathrm{~h}, 3 \mathrm{~h}, 4 \mathrm{~h}, 6 \mathrm{~h}, 8 \mathrm{~h}, 12 \mathrm{~h}$, $24 \mathrm{~h}, 30 \mathrm{~h}, 48 \mathrm{~h}$ after last dosing.

PD sampling

PD samples will be collected at baseline ( -1 day) and 8 days after administration.

\section{Detection method of PK parameter}

The concentration of picrinine, vallesamine, scholaricine and 19-epischolaricinein plasma will be quantitatively analyzed with validated HPLC-MS/MS method.

Detection method of PD parameter

The indexes of SOD, MDA, IgE, Eotaxin, IL-4, IL-10, HMOX1, PDE4B, and PDE4D will be detected by enzyme-linked immune sorbent assay (ELISA) kits, and the ALB will be detected by blood biochemistry.

Proposed trial outcome measures

Primary outcome

(1) Cough disappearance time (clinical efficacy index):

The cough disappearance time of each participant will be recorded to compare the efficacy.

(2) Change in main ingredients of CALAS at different blood concentrations (PK index):

The plasma concentration of four ingredients of picrinine, vallesamine, scholaricine, 19-epischolaricine of theCALAS will be quantitated by HPLC-MS/MS.

Secondary Outcome

(1) The variations of Bronchitis severity score (BSS), Cough symptom score (CSS), and visual analogue scale (VAS) on day 8, compared with the baseline (Clinical efficacy index).

The BSS, CSS and VAS will be recorded at baseline and the $8^{\text {th }}$ day after administration by respiratory physicians. 


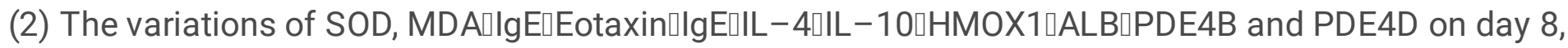
compared with that in baseline (PD index).

The expression of PDE4B and PDE4D, SOD, MDA, IgE, Eotaxin, IL-4, IL-10, HMOX1 enzymes at different time points are detected by ELISA. The content of ALB is determined by serum biochemistry. Moreover the relationship between these indexes and drug concentration will be analyzed.

\section{Safety observation}

Routine laboratory tests include hemograms, biochemical indexes (AST, ALT, ALP, GGT, TBIL, DBIL, IBIL, GLU, BUN), urine routine ECG, and urine pregnancy test for women of childbearing age. Analyzing laboratory tests before and after the test for abnormalities, clinical significance, and test drug relevance.

Covariate: Age, BMI, gender, combination, CL, AST, ALT, ALP, BUN, CREA, GGT, TBIL, HB

PD indexes: SOD, MDA, IgE, Eotaxin, IL-4, IL-10, and ALB, HMOX1, PDE4B and PDE4D which were key targets predicted by network pharmacology.

Metabolomics: The blood samples will be detected to explore the characteristic biomarkers embodying endogenous compounds change before and after administration

Adverse events

Each participant will receive safety monitoring throughout the trial. All adverse events (AE) will be recorded in CRF and the correlation between $\mathrm{AE}$ and CALAS will be judged by the investigators. The serious adverse event will be recorded in SAE form, and reported to the Ethics Committee as soon as possible.

Based on previous studies, the expected adverse reactions of CALAS include hiccup, thirst, nausea, excessive sleeping, abdominal distension, increase bilirubin. And the physicians will try their best to prevent and treat AE which brings by CALAS.

Statistical analysis

Primary efficacy index

Cough disappearance time, selecting group as fixed effect and taking baseline value (age, BMI, gender, combination, CL, AST, ALT, ALP, BUN, CREA, GGT, TBIL, HB) as covariates, will be compared between groups by analysis of covariance method.

Secondary efficacy index

The variation of Bronchitis severity score (BSS), Cough symptom score (CSS), visual analogue scale (VAS) and PD indexes after 7 day dosing, compared with that at baseline are analyzed with ANCOVA method. 
Development of Population Pharmacokinetic/pharmacodynamic model[ ${ }^{0-15]}$

\section{Structure model}

One- two- and three- compartmental models are applied to fit the time data of blood concentrations. A proper model will be selected to characterize the drug in vivo behavior according to the objective function value (OFV) and goodness-of-fit plots. The concentration-time data were fitted using the nonlinear mixedeffects modeling software Phoenix NLME (Certara, Inc., Princeton, New Jersey, USA). The first-order conditional estimation method with the $\eta-\bigotimes(\eta$ : random inter-individual variability; $\Downarrow$ : random residual variability) interaction option (FOCE-ELS) was used throughout the model development process.

\section{Inter-individual variation model}

The exponential model will be applied to characterize the inter-individual variability (IIV) of population pharmacokinetic parameters:

$P_{i}=P \cdot e^{\eta i}$ Equation 1

where $P_{i}$ and $P$ respectively represent the $t^{\text {th }}$ individual value and the typical value for the pharmacokinetic parameter. The relationship between the $i^{\text {th }}$ individual and the population values is described using $\eta_{i}$, which is normally distributed with a mean of zero and a variance of $\omega^{2}$.

\section{Intra-individual variation model}

The residual error is described by the multiplicative error model:

$\mathrm{C}_{\mathrm{i}}=\mathrm{C} \cdot\left(1+\varepsilon_{i)}\right.$ Equation 2

where $C_{i}$ and $C$ accounts for observation and prediction, respectively. $\varepsilon_{i}$ means the residual error of the predicted value, which is normally distributed with zero mean and a variance of $\sigma^{2}$.

\section{Population pharmacokinetic model}

The subject's covariates (Age, BMI, gender, combination, CL, AST, ALT, ALP, BUN, CREA, GGT, TBIL, HB) will be analyzed in a stepwise fashion to identify their potential influences on pharmacokinetic parameters. The categorical covariates (gender and combination) are incorporated using indicator variables. All others (continuous covariates) are centered at the median values and will be included in the model in linear ways. The final PPK is established using the forward inclusion-backward elimination approach. A covariate is considered significant when the addition of this covariate resulted in a decrease in the OFV of $>6.635(P<0.01)$, and an elimination of this covariate resulted in an increase in the OFV of $>10.828(P<$ $0.001)$.

Population Pharmacokinetic/Pharmacodynamic model 
Based on pharmacological action of Alkaloids on the SOD, MDA, IgE, Eotaxin, IL-4, IL-10, and ALB, HMOX1, PDE4B and PDE4D, the relationship between blood drug concentration and PD indexes will fit using an integral PPK/PD model. The indirect response (IDR) model will be used to develop pharmacodynamic model. All the PD indexes are produced with a zero-order input rate and dissipated with a first-order output rate. The rate of change of the response over time with no drug present can be described by

$\mathrm{dR} / \mathrm{dt}=\mathrm{K}_{\text {in }}-\mathrm{K}_{\text {out }} \cdot \mathrm{R}$ Equation 4

where $\mathrm{R}$ is the measured PD index, $\mathrm{K}_{\text {in }}$ represents the zero-order constant for production of the response and $\mathrm{K}_{\text {out }}$ defines the first-order rate constant for loss of the response. It is assumed that $\mathrm{K}_{\text {in }}$ and $\mathrm{K}_{\text {out }}$ fully account for production and loss of the response.

According to the action mechanism, drug concentration may stimulate (Equation 7 and 8) or inhibit (Equation 9 and 10) the PD indexes

$S(t)=1+E_{\max } \cdot C /\left(E_{50}+C\right)$ Equation 5

$I(t)=1-C /\left(C+I C_{50}\right)$ Equation 6

where $\mathrm{C}$ is the drug concentration. $E_{\max }$ represents the maximum effect attributed to the drug and $E C_{50}$ represents drug concentration producing $50 \%$ of the maximum stimulation achieved at the effect site. $\mathrm{IC}_{50}$ is the drug concentration which produces $50 \%$ of maximum inhibition achieved at the effect site

$\mathrm{dR} / \mathrm{dt}=\mathrm{K}_{\text {in }} \mathrm{S}(\mathrm{t})-\mathrm{K}_{\text {out }} \cdot \mathrm{R}$ Equation 7

$\mathrm{dR} / \mathrm{dt}=\mathrm{K}_{\text {in }}-\mathrm{K}_{\text {out }} \cdot \mathrm{S}(\mathrm{t}) \cdot \mathrm{R}$ Equation 8

$\mathrm{dR} / \mathrm{dt}=\mathrm{K}_{\text {in }} \mathrm{I}(\mathrm{t})-\mathrm{K}_{\text {out }} \cdot \mathrm{R}$ Equation 9

$\mathrm{dR} / \mathrm{dt}=\mathrm{K}_{\text {in }}-\mathrm{K}_{\text {out }} \cdot \mathrm{I}(\mathrm{t}) \cdot \mathrm{R}$ Equation 10

\section{Model verification}

Model validation checks the accuracy of the model's representation of the observation through bootstrap and visual predictive check (VPC). A bootstrap method involved repeated re-sampling with a replacement from the measured data for 1000 times, and the final model will be fitted to the bootstrap datasets. The values of bootstrap parameters [median and the $95 \%$ confidence intervals (Cls)] are compared with the parameters of final PPK/PD model. The VPC uses 1000 times Monte Carlo simulation to generate concentration-time profiles. To evaluate the predictive performance of the final PPK/PD model, the observed data will compare with the $5^{\text {th }}, 50^{\text {th }}$, and $95^{\text {th }}$ percentiles of the simulated data. 
Interim analysis will not perform in this study.

\section{Discussion}

Traditional Chinese medicine (TCM) characterized by "multi-compound and weak-effect" is different from western medicine. TCM consists of many component which have low biological activity, and the overall efficacy of it is not simply the efficacy accumulation by the single component, but a multi-level, multi-link and multi-dimensional nonlinear effects ${ }^{[16]}$. In view of TCM characteristics of "multi-component and multi-efficacy", the current researches on the mechanism and target of TCM have shown a transformation trend from a single mechanism or pathway to comprehensive mechanism and multipathway ${ }^{[17-18]}$. In recent years, it turns out a variety of strategies for target prediction, identification and verification, with the development of bioinformatics and computer science. Network pharmacology, as one of the best recommended strategies, is highly consistent with holism of TCM since its integrality and systematicness, and could reflect the characteristics of multi-component, multi-pathway and multi-target in $\mathrm{TCM}^{[19]}$. Although the research on the complex mechanism of TCM based on network pharmacology has become a hot topic, however it is mostly concentrated in the preclinical stage, which has not been further verified by clinical data based on the human samples and clinical factors ${ }^{[20-21]}$. Furthermore, strategies for evaluating the correlation between targets or pathways predicted by network pharmacology, and clinical effect have not been established.

In addition, the quantitative pharmacology method, another way to explore mechanism of TCM, is to conduct "dose-exposure" model research. As we know, the effectiveness of drug depends on blood concentration. Modern research indicates that the composition of active components and the blood concentration are tightly correlated with the effects of specific pathways or targets in the multicomponent pharmacology network ${ }^{[22]}$. Therefore, the resarch of pharmacokinetics (PK) and pharmacodynamics (PD) evaluation model research is not only contributed to analyzing the dynamic process of drug absorption, distribution, metabolism and excretion in vivo, but also preliminarily evaluating the clinical efficacy of TCM. However, there are still two bottlenecks in the PK-PD study of TCM, one is difficult to identify the PK markers which have really acted in the complex component system of TCM, and the other is difficult to find PD biomarkers which have the strongest association with efficacy and can sensitively reflect the drug exposure level in vivo.

Hence, the research tries to establish a new integrating strategy to explore relationship among drug ingredients, action pathway and clinical efficacy aiming at the existing problems of network pharmacology and quantitative pharmacology. On the premise of definite active ingredients and reliable clinical efficacy, the purpose of the study is to explore the mechanism of CALAS in treatment of acute bronchitis. It is difficult to be accepted by patients since traditional PK research adopts intensive sampling method, meanwhile, it cannot quantify the variability of the pharmacokinetic parameters (intraindividual variation, interindividual variation and weekly variation). Moreover, the extrapolation and prediction of dosage regimen between different species and populations cannot be realized ${ }^{\text {[23] }}$. 
Therefore, pharmacokinetics (PK) and pharmacodynamics (PD) method, taking advantage of sparse data, (usually 3-5 time points sampling per patient), will be selected to find out the measurable factors of pathology and physiology that lead to the change of dose-concentration, and guide reasonable dose adjustment to achieve the optimal clinical effects ${ }^{[24]}$.

\section{Trial Status}

Recruitment began January 2019.

\section{Abbreviations}

CALAS,capsule of alkaloids from leaf of Alstonia scholaris; PPK/PD,population pharmacokinetics/pharmacodynamics; TCM,Traditional Chinese medicine; Cls,confidence intervals; ALB,Serum albumin; AR,Androgen receptor; GSTP1,Glutathione S-transferase P; HMOX1,Heme oxygenase 1;MMP13,Collagenase 3; KDR, Vascular endothelial growth factor receptor 2; PDE4B, cAMP-specific 3,5cyclic phosphodiesterase 4B; PDE4D,cAMP-specific 3,5-cyclic phosphodiesterase 4D; BSS,Bronchitis severity score; CSS,Cough symptom score; VAS,visual analogue scale.

\section{Declarations}

\section{Ethics approval and consent to participate}

Institutional ethics approval for the trial was obtained from the Ethics Committee of Xiyuan Hospital. The protocol number is version No.1.1 Version date is 2018-05-18. Informed consent will be obtained from all participants. The investigators and respiratory physicians will keep the confidentiality of the data, according to the privacy rules of clinical practice. Only authorized investigators have access to the final trial data set. Patients will be asked for permission for data analysis and publish in an anonymous way.

\section{Consent for publication}

Not applicable.

\section{Availability of data and materials}

The SPIRIT checklist has been completed and made available.

\section{Competing interests}

The authors declare that they have no competing interests. 


\section{Funding}

The study is supported by National Science of China Academy of Chinese Medical Sciences (No. zz0908022). National natural science foundation of China (81603505), National science and technology major project (2017ZX09304003).

\section{Authors' contributions}

Rui Li conceived the study and developed the first trial protocol, Wan-Tong Zhang designed the study and drafted the manuscript, Mao-Rong Fan participated in the subject selection criteria setting, Yun-Li Zhao provided the preclinical pharmacodynamic data and revise the manuscript, Ming-Yue Sun provided the network prediction result, xin-Gang Li provided the PPK-PPD analysis method, Xiao-Dong Luo and Rui Gao contributed to the editing of the final manuscript and approved the final version.

\section{Trial status}

Recruitment began in Jan 2019 and will complete in Oct 2019.

The protocol number is version No.1.1 Version date is 2018-05-18.

\section{Additional file}

Additional file 1: SPIRIT 2013 checklist. (DOC 114 kb)

Additional file 2: SPIRIT Figure.( JPG 33kb)

\section{Reference}

[1]Scott kinkade, Nataline A. Long. Acute Bronchitis. American Family Physician.2016, 94(7):560-565

[2]Yun-LiZhao, Jian-HuaShang, Shi-BiaoPu, Heng-Shan Wang, Bei Wang, Lu Liu, Ya-ping Liu, Hong-Mei Shen, Xiao-Dong Luo. Effect of total alkaloids from Alstonia scholaris on airway inflammation in rats[J]. Journal of Ethnopharmacology, 2016,178:258-265.

[3]Jian-Hua Shang, Xiang-Hai Cai, Tao Feng, et al. Pharmacological evaluation of Alstonia scholaris: Antiinflammatory and analgesic effects[J]. Journal of Ethnopharmacology,129 (2010):174-181.

[4]Jian-Hua Shang, Xiang-Hai Cai, Yun-Li Zhao, et al. Pharmacological evaluation of Alstonia scholaris: Anti-tussive, anti-asthmatic and expectorant activities[J]. Journal of Ethnopharmacology, 129 (2010):293-298. 
[5]Yuanyuan Hou, Xuelin Cao, Liqiang Wang, Bingfeng Cheng, Linyi Dong, Xiaodong Luo, Gang Bai, Wenyuan Gao. Microfractionation bioactivity-based ultra performance liquid chromatography/quadrupole time-of-flight mass spectrometry for the identification of nuclear factor-_B inhibitors and _2 adrenergic receptor agonists in an alkaloidal extract of the folk herb Alstonia scholaris[J]. Journal of Chromatography B, 908 (2012):98- 104.

[6]Benkali K, Rostaing L, Premaud A, er al. Population pharmacokinetics and Bayesian estimation of tacrolimus exposure in renal transplant recipients on a new once-daily formulation. Clin Pharmacokinet,2010, 49: 683-692 .

[7]Wang Dongdong, Lu Jinmiao, Li Qin, et al. Population pharmacokinetics of tacrolimus in pediatric refractory nephrotic syndrome and a summary of other pediatric disease models[J]. Exp Ther Med,2019. $17,4023-4031$.

[8]Willavize S, Fiedler-Kelly J, Ludwig E, et al. Population Pharmacokinetic Modeling of Armodafinil and Its Major Metabolites[J].J Clin Pharmacol,2017,57(2):255-265.

[9]Sun mingyue, gao rui, lu fang, et al. Mechanism of dengtai total alkaloid capsules in the treatment of acute bronchitis based on network pharmacology. New Chinese medicine and clinical pharmacology, 2018, 29 (4) :468-473.

[10]Benkali Khaled, Prémaud Aurelie,Picard Nicolas, et al. Tacrolimus population pharmacokineticpharmacogenetic analysis and Bayesian estimation in renal transplant recipients.[J] .Clin Pharmacokinet, 2009, 48: 805-16.

[11]Antignac $M$, Barrou B, Farinotti R, Lechat $P$, Urien $S$. Population pharmacokinetics and bioavailability of tacrolimus in kidney transplant patients[J]. British Journal of Clinical Pharmacology. 2007; 64(6):7507.

[12]Zimmermann ES, Laureano JV, Dos Santos CN,et al. Simultaneous Semimechanistic Population Analyses of Levofloxacin in Plasma, Lung, and Prostate To Describe the Influence of Efflux Transporters on Drug Distribution following Intravenous and Intratracheal Administration[J].Antimicrob. Agents Chemother. 2016,60(2): 1315-17.

[13]Anderson BJ, Holford NH. Mechanism-based concepts of size and maturity in pharmacokinetics [J]. Annu. Rev. Pharmacol. Toxicol 2008; 48:303-32.

[14]Prytuła AA, Cransberg K, Bouts AH, van Schaik RH, de Jong H, de Wildt SN, et al. The Effect of Weight and CYP3A5 Genotype on the Population Pharmacokinetics of Tacrolimus in Stable Paediatric Renal Transplant Recipients [J]. Clinical Pharmacokinetics 2016; 55(9):1-15.

[15]Nguyen TH, Mouksassi MS, Holford N, Al-Huniti N, Freedman I, Hooker AC, et al. Model Evaluation of Continuous Data Pharmacometric Models: Metrics and Graphics[J].CPT Pharmacometrics Syst 
Pharmacol. 2017,6(2):87-109.

[16] Li Tan, Baochen Zhu, Yue Zhang et al. Network Pharmacology-Based identification of pharmacological mechanism of SQFZ injection in combination with Docetaxel on lung cancer.[J] .Sci Rep, 2019, 9: 4533-4537.

[17] Feng liang, zhang minghua, gu junfei, et al. Innovation and practice of the "component structure" theory of the material basis of traditional Chinese medicine. Chinese journal of traditional Chinese medicine, 2013,38 (21) : 3603-3607.

[18] Xiao Mei-Feng,Duan Xiao-Peng,Deng Kai-Wen et al. [Dynamic chromatopharmacokinetics model for components in Chinese medicine and validation in Buyang Huanwu Decoction].[J] .Zhongguo Zhong Yao Za Zhi, 2019, 44: 574-581.

[19] Zhu Jiayi,Shen Lan,Lin Xiao et al. Clinical Research on Traditional Chinese Medicine compounds and their preparations for Amyotrophic Lateral Sclerosis.[J] .Biomed. Pharmacother., 2017, 96: 854-864.

[20] Wang Jigang,Wong Yin-Kwan,Liao Fulong,What has traditional Chinese medicine delivered for modern medicine?[J] .Expert Rev Mol Med, 2018, 20: e4.

[21] Han Shuxian,Chen Ying,Wang Jinyu et al. Anti-thrombosis Effects and Mechanisms by Xueshuantong Capsule Under Different Flow Conditions.[J] .Front Pharmacol, 2019, 10: 35.

[22]Zhu Baochen, Zhang Wantong, Lu Yang, et al. Network pharmacology-based identification of protective mechanism of Panax Notoginseng Saponins on aspirin induced gastrointestinal injury.[J] .Biomed. Pharmacother., 2018, 105: 159-166.

[23] Wang Dongdong,Lu Jinmiao,Li Qin et al. Population pharmacokinetics of tacrolimus in pediatric refractory nephrotic syndrome and a summary of other pediatric disease models.[J] .Exp Ther Med, 2019, 17: 4023-4031.

[24] Guidi Monia,Mercier Thomas,Aouri Manel et al. Population pharmacokinetics and pharmacodynamics of the artesunate-mefloquine fixed dose combination for the treatment of uncomplicated falciparum malaria in African children.[J] .Malar. J., 2019, 18: 139.

\section{Table 1}

Table 1 Study diagram 


\begin{tabular}{|c|c|c|c|c|}
\hline & D-14-D-1 & D1-D7 & D8 & D9-D10 \\
\hline Informed consent & $x$ & & & \\
\hline Demographic information & $x$ & & & \\
\hline General questions, medical history & $x$ & & $x$ & \\
\hline Physical examination & $x$ & & & \\
\hline ECG & $x$ & & $x$ & \\
\hline Vital signs & $x$ & & $x$ & \\
\hline $\begin{array}{l}\text { Laboratory examination (blood routine, blood biochemistry, urine } \\
\text { routine, urine HCG) }\end{array}$ & $x$ & & $x$ & \\
\hline X-Ray & $x$ & & & \\
\hline PD indexes & $x$ & & $x$ & \\
\hline Clinical symptoms & $x$ & & $x$ & \\
\hline Randomize & $x$ & & & \\
\hline Administration & & $x$ & $x$ & \\
\hline PK sampling & & $x$ & $x$ & $x$ \\
\hline $\mathrm{AE}$ & & $x$ & $x$ & $x$ \\
\hline Drug combination & $x$ & $x$ & $x$ & $x$ \\
\hline
\end{tabular}

\section{Supplementary Files}

This is a list of supplementary files associated with this preprint. Click to download.

- supplement1.jpg

- supplement2.doc 